\title{
Gluon masses without seagull divergences
}

\section{Joannis Papavassiliou}

Department of Theoretical Physics and IFIC,

University of Valencia-CSIC,

E-46100, Valencia, Spain.

E-mail: Joannis.Papavassiliou@uv.es

The study of dynamical gluon mass generation at the level of Schwinger-Dyson equation involves a delicate interplay between various field-theoretic mechanisms The underlying local gauge invariance remains intact by resorting to the well-known Schwinger mechanism, which is assumed to be realized by longitudinally coupled bound state poles, produced by the non-perturbative dynamics of the theory. These poles are subsequently included into the Schwinger-Dyson equation of the gluon propagator through the three-gluon vertex, generating a non-vanishing gluon mass, which, however, is expressed in terms of divergent seagull integrals. In this talk we explain how such divergences can be eliminated completely by virtue of a characteristic identity, valid in dimensional regularization. The ability to trigger this identity depends, in turn, on the details of the three-gluon vertex employed, and in particular, on the exact way the bound state poles are incorporated. A concrete example of a vertex that triggers the aforementioned identity is constructed, the ensuing cancellation of all seagull divergences is explicitly demonstrated, and a finite gluon mass is obtained. Due to the multitude of conditions that must be simultaneously satisfied, this construction appears to be exclusively realized within the PT-BFM framework. The resulting system of integral equations gives rise to a gluon mass that displays power-law running and an effective charge which, due to the presence of the gluon mass, freezes in the infrared at a finite (non-vanishing) value.

International Workshop on QCD Green's Functions, Confinement, and Phenomenology - QCD-TNT09 September 07 - 112009

ECT Trento, Italy 


\section{Introduction}

The gluon is massless at the level of the fundamental QCD Lagrangian, and remains massless to all order in perturbation theory. However, as Cornwall argued in the early eighties [1], the non-perturbative QCD dynamics generate an effective, momentum-dependent mass for the gluons, without affecting the local $S U(3)_{c}$ invariance, which remains intact.

Given that the gluon mass generation is a purely non-perturbative effect, the natural framework to study it, in the continuum, are the Schwinger-Dyson equations (SDEs) of the theory. At the level of the SDEs the generation of such a mass is associated with the existence of infrared finite solutions for the gluon propagator $[1,2,3]$. In covariant gauges, the gluon propagator, $\Delta_{\mu v}(q)$, has the form

$$
\Delta_{\mu v}(q)=-i\left[\mathrm{P}_{\mu v}(q) \Delta\left(q^{2}\right)+\xi \frac{q_{\mu} q_{v}}{q^{4}}\right]
$$

where $\xi$ denotes the gauge-fixing parameter, and $\mathrm{P}_{\mu \nu}(q)=g_{\mu \nu}-q_{\mu} q_{v} / q^{2}$. The scalar factor $\Delta\left(q^{2}\right)$ is given by $\Delta^{-1}\left(q^{2}\right)=q^{2}+i \Pi\left(q^{2}\right)$, where $\Pi_{\mu v}(q)=\mathrm{P}_{\mu v}(q) \Pi\left(q^{2}\right)$ is the gluon self-energy; the dimensionless vacuum polarization, $\Pi\left(q^{2}\right)$, is defined as $\Pi\left(q^{2}\right)=q^{2} \Pi\left(q^{2}\right)$. So, in general, one looks for solutions with $\Delta^{-1}(0)>0$. Such solutions may be fitted by "massive" propagators of the form $\Delta^{-1}\left(q^{2}\right)=q^{2}+m^{2}\left(q^{2}\right) ; m^{2}\left(q^{2}\right)$ is not "hard", but depends non-trivially on the momentum transfer $q^{2}$.

The pinch technique (PT) propagator, usually denoted by $\widehat{\Delta}\left(q^{2}\right)$ in the literature $[1,4]$, is the ideal quantity to study in this context, because it is independent of the gauge-fixing parameter $(\xi)$. Therefore, any statement about its infrared behavior, and in particular the generation of a gluon mass, is bound to be free of gauge artefacts. In recent studies, however, the tendency has been to focus on the gluon propagator in a fixed gauge, such as the conventional Landau gauge $(\xi=0)$, instead of the privileged Feynman gauge $\left(\xi_{Q}=1\right)$ of the background field method (BFM) [5], which, quite remarkably, reproduces automatically the PT results (third item in [4]). The main reason for this less optimal choice is the need to compare meaningfully the SDE results with those obtained from lattice studies, which, almost exclusively, are carried out in the Landau gauge.

What the latest large-volume lattice studies reveal is crystal clear: The gluon propagator of pure Yang-Mills is infrared finite, both in $S U(2)$ [6] and $S U(3)$ [7]. Interestingly enough, these recent lattice findings, striking as they may be, do not constitute the first indication of this very characteristic behavior; several earlier simulations had found qualitatively similar results, even in gauges other than the Landau ( see, e.g., [8]).

The aforementioned lattice results, in addition to whatever modifications they may induce to other formalisms aspiring to describe the infrared sector of QCD, they present a serious challenge even to the practitioners of the gluon mass generation (albeit, a pleasant one). Indeed, the SDE analysis must be further refined, and freed of whatever major or minor theoretical shortcomings one has been accustomed to live with in the past. The purpose of this talk is to report recent progress in this direction, and in particular on the solution of the annoying problem of seagull divergences [9], which has afflicted this approach from the first day of its invention [1]. Turns out that the elimination of the seagull divergences brings about an additional advantage, namely the separation of the SDE into two coupled equations, furnishing uniquely the running of the effective charge and the gluon mass in the entire range of Euclidean momenta. 


\section{Seagull divergences: a perennial nuisance to gluon mass generation}

In order to obtain massive solutions gauge-invariantly, it is necessary to invoke the well-known Schwinger mechanism [10]. The basic observation is that if, for some reason, $\Pi\left(q^{2}\right)$ acquires a pole at zero momentum transfer, then the vector meson becomes massive, even if the gauge symmetry forbids a mass at the level of the fundamental Lagrangian. Indeed, it is clear that if the vacuum polarization $\Pi\left(q^{2}\right)$ has a pole at $q^{2}=0$ with positive residue $\mu^{2}$, i.e. $\Pi\left(q^{2}\right)=\mu^{2} / q^{2}$, then (in Euclidean space) $\Delta^{-1}\left(q^{2}\right)=q^{2}+\mu^{2}$. Thus, the vector meson becomes massive, $\Delta^{-1}(0)=\mu^{2}$, even though it is massless in the absence of interactions $(g=0)$. There is no physical principle which would preclude $\Pi\left(q^{2}\right)$ from acquiring such a pole. In a strongly-coupled theory like QCD this may happen for purely dynamical reasons, since strong binding may generate zero-mass bound-state excitations [11]. The latter act like dynamical Nambu-Goldstone bosons, in the sense that they are massless, composite, and longitudinally coupled; but, at the same time, they differ from NambuGoldstone bosons as far as their origin is concerned: they do not originate from the spontaneous breaking of any global symmetry [1].

Of course, in order to obtain the full dynamics, such as, for example, the momentum-dependence of the dynamical mass, one must turn eventually to the SDE that governs the corresponding gaugeboson self-energy. The way the Schwinger mechanism is integrated into the SDE is through the form of the three-gluon vertex. The latter, even in the absence of mass generation, constitutes a central ingredient of the SDE, and plays a crucial role in enforcing the transversality of the gluon self-energy. Therefore, an important requirement for any self-consistent Ansatz used for that vertex is that it should satisfy the correct Ward identity (WI) of the PT-BFM formulation, namely

$$
q^{\mu} \widetilde{\Gamma}_{\mu \alpha \beta}=\Delta_{\alpha \beta}^{-1}(k+q)-\Delta_{\alpha \beta}^{-1}(k) .
$$

In addition, in order to generate a dynamical mass one must assume that the vertex contains $d y$ namical poles, which will trigger the Schwinger mechanism when inserted into the SDE for the gluon self-energy.

The point is that the full realization of the procedure outlined above is very subtle. In particular, even though the use of a three-gluon vertex containing massless poles and satisfying the correct WI leads indeed to a transverse and infrared finite self-energy (i.e. $\Delta^{-1}(0) \neq 0$ ), as expected, the actual value of $\Delta^{-1}(0)$ has always been expressed in terms of divergent integrals, of the form (see, e.g., $[1,2,3])$

$$
\Delta^{-1}(0)=c_{1} \int_{k} \Delta(k)+c_{2} \int_{k} k^{2} \Delta^{2}(k),
$$

where, in dimensional regularization (DR), $\int_{k} \equiv \mu^{2 \varepsilon}(2 \pi)^{-d} \int d^{d} k$, with $d=4-\varepsilon$ the dimension of space-time. This is not a problem, in principle, provided that the divergent integrals appearing on the rhs of (2.2) can be properly regulated and made finite, without introducing counterterms of the form $m_{0}^{2}\left(\Lambda_{\mathrm{UV}}^{2}\right) A_{\mu}^{2}$, which are forbidden by the local gauge invariance of the fundamental QCD Lagrangian. Various regularization procedures of increasing sophistication have been tried out over the years, but the resulting (regularized) $\Delta^{-1}(0)$ remained theoretically ambiguous. Given how nicely all other pieces of the puzzle fit together, the underlying impression has always been that the "seagull problem" had to do with some (not fully understood) subtlety rather than some intrinsic "need" of the theory to produce quadratic divergences. 

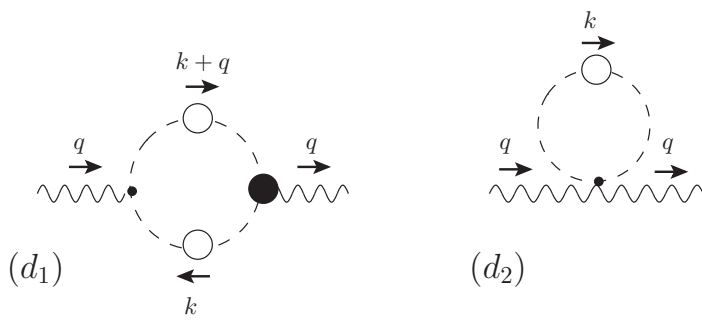

$\left(d_{2}\right)$

Figure 1: The "one-loop dressed" SDE for the photon self-energy.

\section{The "seagull identity": how to keep the photon massless (if you must)}

It is most instructive to understand what happens in a theory where the seagull terms do not appear due to the self-interactions of a gauge boson that has acquired a mass dynamically, but rather because the theory has scalar particles that are massive at tree-level. These scalars interact with the gauge boson, and contribute seagull terms to its vacuum polarization. The question is: if the gauge boson must remain massless, how do the seagull contributions disappear from that vacuum polarization? To see how this happens, let us turn to scalar QED, where the aforementioned circumstances (massive scalars, must-be massless photon) are realized, and study the SDE governing the photon self-energy.

At the "one-loop dressed" level the SDE for the photon self-energy reads (Fig. 1)

$$
\Pi_{\mu v}(q)=e^{2} \int_{k} \Gamma_{\mu}^{(0)} \mathscr{D}(k) \mathscr{D}(k+q) \Gamma_{v}+e^{2} \int_{k} \Gamma_{\mu \nu}^{(0)} \mathscr{D}(k),
$$

where $\mathscr{D}(k)$ is the fully-dressed propagator of the scalar field. $\Gamma_{v}$ is the fully dressed photonscalar vertex, whose tree-level expression is given by $\Gamma_{\mu}^{(0)}=-i(2 k+q)_{\mu}$. Moreover, the bare quatrilinear photon-scalar vertex is given by $\Gamma_{\mu \nu}^{(0)}=2 i g_{\mu \nu}$. The photon-scalar vertex $\Gamma_{\mu}$ and the scalar propagator $\mathscr{D}$ are related by the Abelian all-order WI

$$
q^{v} \Gamma_{v}=\mathscr{D}^{-1}(k+q)-\mathscr{D}^{-1}(k)
$$

It is fairly easy to demonstrate that, by virtue of (3.2), $q^{v} \Pi_{\mu v}(q)=0$, and that $\Pi\left(q^{2}\right)$ reads

$$
\Pi\left(q^{2}\right)=\frac{-2 i e^{2}}{d-1}\left[\int_{k} \mathscr{D}(k) \mathscr{D}(k+q) k^{\mu} \Gamma_{\mu}-d \int_{k} \mathscr{D}(k)\right],
$$

Let us compute from (3.3) the one-loop expression for $\Pi\left(q^{2}\right)$, to be denoted by $\Pi^{(1)}\left(q^{2}\right)$.

$$
\Pi^{(1)}\left(q^{2}\right)=\frac{-i e^{2}}{d-1}\left[\int_{k}\left(4 k^{2}-q^{2}\right) \mathscr{D}_{0}(k) \mathscr{D}_{0}(k+q)-2 d \int_{k} \mathscr{D}_{0}(k)\right],
$$

where $\mathscr{D}_{0}(k)=\left(k^{2}-m^{2}\right)^{-1}$. Taking the limit $q \rightarrow 0$, we find

$$
\Pi^{(1)}(0)=\frac{-4 i e^{2}}{d-1}\left[\int_{k} k^{2} \mathscr{D}_{0}^{2}(k)-\frac{d}{2} \int_{k} \mathscr{D}_{0}(k)\right] .
$$

Of course, there is no doubt that the photon remains massless perturbatively, i.e. we must have that $\Pi^{(1)}(0)=0$. However, the way this requirement is realized is rather subtle: the rhs of (3.5) 
vanishes indeed, by virtue of an identity that is exact in DR, namely

$$
\int_{k} \frac{k^{2}}{\left(k^{2}-m^{2}\right)^{2}}=\frac{d}{2} \int_{k} \frac{1}{k^{2}-m^{2}},
$$

Thus, the perturbative masslessness of the photon is explicitly realized and self-consistently enforced within the DR. Eq.(3.6) may be cast in a form that is particularly suggestive for the analysis that follows, namely

$$
\int_{k} k^{2} \frac{\partial \mathscr{D}_{0}(k)}{\partial k^{2}}=-\frac{d}{2} \int_{k} \mathscr{D}_{0}(k) .
$$

We now return to the general Eq.(3.3). In order to analyze it further we must furnish some information about the form of $\Gamma_{\mu}$. Of course, any meaningful Ansatz for $\Gamma_{\mu}$ must satisfy the WI of (3.2), or else the transversality of $\Pi_{\mu v}(q)$ will be compromised from the outset. The form obtained by Ball and Chiu [12], after "solving" the WI, under the additional requirement of not introducing kinematic singularities, is (we omit the identically conserved part of the vertex)

$$
\Gamma_{\mu}=\frac{(2 k+q)_{\mu}}{(k+q)^{2}-k^{2}}\left[\mathscr{D}^{-1}(k+q)-\mathscr{D}^{-1}(k)\right] .
$$

This vertex, when substituted into (3.3), yields

$$
\Pi\left(q^{2}\right)=\frac{i e^{2}}{d-1}\left[\int_{k}\left(4 k^{2}-q^{2}\right) \frac{\mathscr{D}(k+q)-\mathscr{D}(k)}{(k+q)^{2}-k^{2}}+2 d \int_{k} \mathscr{D}(k)\right] .
$$

Taking the limit of Eq.(3.9) as $q \rightarrow 0$, using that

$$
\frac{\mathscr{D}(k+q)-\mathscr{D}(k)}{(k+q)^{2}-k^{2}} \rightarrow \frac{\partial \mathscr{D}(k)}{\partial k^{2}}+\mathscr{O}\left(q^{2}\right),
$$

we have that

$$
\Pi(0)=\frac{4 i e^{2}}{d-1}\left[\int_{k} k^{2} \frac{\partial \mathscr{D}(k)}{\partial k^{2}}+\frac{d}{2} \int_{k} \mathscr{D}(k)\right],
$$

Of course, we must have that $\Pi(0)=0$, given that there is nothing in the dynamics that could possibly endow the photon with a mass; in particular, the Schwinger's mechanism is "switched off", i.e. we have not introduced dynamical poles, and, given the form of (3.8), neither kinematic ones, which might simulate the dynamical ones at the level of the SDE. Thus, the rhs of (3.11)must vanish, and therefore, we must have that

$$
\int_{k} k^{2} \frac{\partial \mathscr{D}(k)}{\partial k^{2}}=-\frac{d}{2} \int_{k} \mathscr{D}(k)
$$

which is the non-perturbative generalization of (3.7).

Note a crucial point: the seagull terms appearing in (3.7) cannot be set to zero individually, because the scalar propagator inside them is massive (already at tree-level): the only way to keep the photon massless, is to employ (3.7), which cancels them against each other. For example, if the term $\int_{k} \mathscr{D}(k)$ on the rhs were multiplied by any factor other than $(d / 2)$ one would be stuck with seagull divergences. 

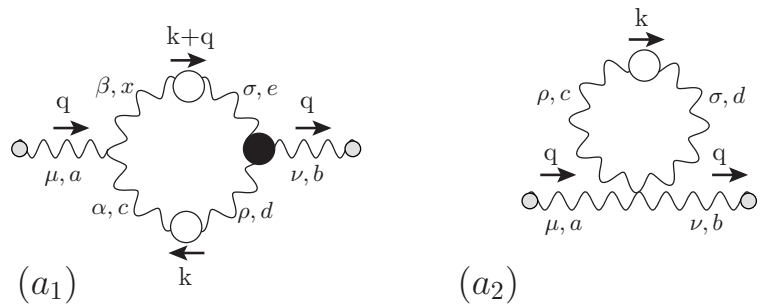

Figure 2: "One-loop dressed" gluonic graphs of the SDE for the PT-BFM gluon self-energy.

\section{Massive gluons: Schwinger mechanism and seagull identity in a delicate balance}

Turns out that the construction presented in the previous section generalizes in the context of pure Yang-Mills theory, such as quarkless QCD, but only within the PT-BFM formalism! As has been explained in detail in the recent literature [2,13], this latter formalism allows for a gaugeinvariant truncation of the SD series, in the sense that it preserves manifestly and at every step the transversality of the gluon self-energy. Specifically, for the case at hand, we will consider only the "one-loop dressed" part of the gluon SDE that contains gluons, shown in Fig. 2, leaving out (gauge-invariantly!) the "one-loop dressed" ghost contributions and all "two-loop dressed" diagrams. Note that the Feynman rules used to build this SD series are those of the BFM [5]; in particular, the external gluons (distinguished by the grey circles attached to them) are treated as if they were background gluons. As we will see in a moment, the form of these vertices is crucial for obtaining from the SDE precisely the right combination of terms (and with the correct relative weights) that appears in (3.12).

In order to reduce the algebraic complexity of the problem, we drop the longitudinal terms from the gluon propagators inside the integrals, i.e. we set $\Delta_{\alpha \beta} \rightarrow-i g_{\alpha \beta} \Delta$. This does not compromise the transversality of $\widehat{\Pi}_{\mu v}(q)$ provided that we do the same on the rhs of the WI satisfied by $\widetilde{\Gamma}_{v \alpha \beta}$, namely we have simply

$$
q^{v} \widetilde{\Gamma}_{v \alpha \beta}=\left[\Delta^{-1}(k+q)-\Delta^{-1}(k)\right] g_{\alpha \beta},
$$

instead of the full WI given in (2.1). Then, the SDE corresponding to Fig. 2 reduces to

$$
\widehat{\Delta}^{-1}(q)=q^{2}+\frac{i g^{2} C_{\mathrm{A}}}{2(d-1)}\left[\int_{k} \widetilde{\Gamma}_{\mu \alpha \beta}^{(0)} \Delta(k) \Delta(k+q) \widetilde{\Gamma}^{\mu \alpha \beta}+2 d^{2} \int_{k} \Delta(k)\right],
$$

where $C_{\mathrm{A}}$ the Casimir eigenvalue of the adjoint representation $\left[C_{\mathrm{A}}=N\right.$ for $\left.S U(N)\right]$. The vertex $\widetilde{\Gamma}_{\mu \alpha \beta}^{(0)}(q, k,-k-q)$ is the bare three-gluon vertex in the Feynman gauge of the BFM, and $\widetilde{\Gamma}_{\mu \alpha \beta}$ denotes its fully-dressed version.

The function $\widehat{\Delta}(q)$ appearing on the lhs of (4.2) is the scalar part of the gluon propagator in the BFM, i.e. two background gluons entering. $\widehat{\Delta}(q)$ is related to the standard $\Delta(q)$, defined in the $R_{\xi}$ gauges, by means of the powerful identity $\widehat{\Delta}(q)\left[1+G\left(q^{2}\right)\right]^{2}=\Delta(q)$, where $G\left(q^{2}\right)$ is an auxiliary two-point function [14], which, quite remarkably, coincides in the Landau gauge with the wellknown Kugo-Ojima function (see, e.g. talk of Daniele Binosi in these proceedings [16]). We will next set $G\left(q^{2}\right)=0$, i.e. we effectively assume that, inside the quantum loops, $\Delta(q)=\widehat{\Delta}(q)$. 


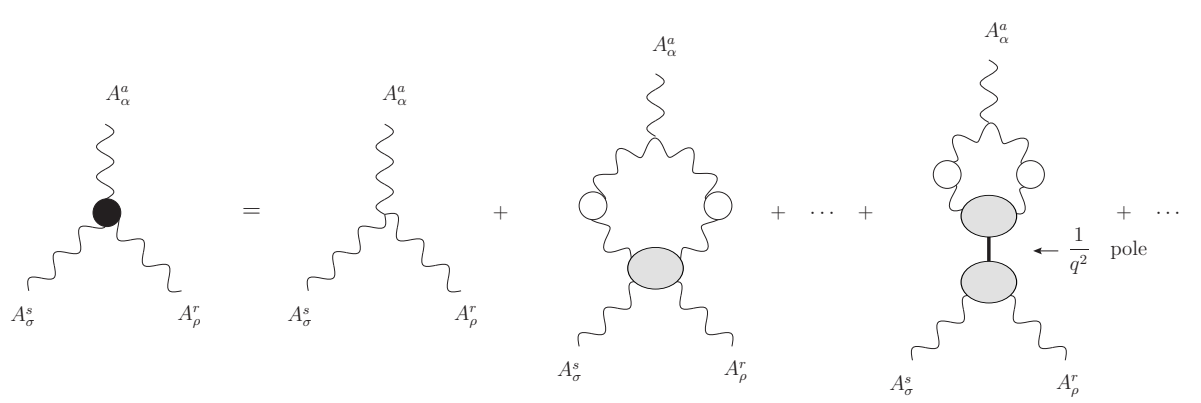

Figure 3: The SDE for the three-gluon vertex. All kernels are one-particle irreducible, and the $1 / q^{2}$ pole is not kinematic but dynamical (purely non-perturbative); physically it corresponds to a (composite) Goldstone mode, necessary for maintaining the local gauge invariance.

At this point enters the new ingredient: a judicious Ansatz for the three-gluon vertex which, in addition to satisfying (4.1) will allow us to use the seagull identity (3.12) and get a non-vanishing and finite $\Delta^{-1}(0)$.

To begin with, let us first write $\Delta^{-1}(q)$ in the alternative form (in Minkowski space)

$$
\Delta^{-1}(q)=q^{2} H^{-1}(q)-\widetilde{m}^{2}(q) .
$$

The tree-level result for $\Delta^{-1}(q)$ is recovered by setting $H^{-1}(q)=1$ and $\widetilde{m}^{2}=0$. Then, an appropriate Ansatz for $\widetilde{\Gamma}_{v \alpha \beta}$ is given by [9]

$$
i \widetilde{\Gamma}_{\mu \alpha \beta}=\left[\frac{(k+q)^{2} H^{-1}(k+q)-k^{2} H^{-1}(k)}{(k+q)^{2}-k^{2}}\right] \widetilde{\Gamma}_{\mu \alpha \beta}^{(0)}+V_{\mu \alpha \beta},
$$

where the term $V_{\mu \alpha \beta}$ contains the non-perturbative contributions due to bound-state poles associated with the Schwinger mechanism. Note that we must have

$$
q^{\mu} V_{\mu \alpha \beta}=\left[\widetilde{m}^{2}(k)-\widetilde{m}^{2}(k+q)\right] g_{\alpha \beta},
$$

in order for the $\widetilde{\Gamma}_{\mu \alpha \beta}$ of Eq. (4.4) to satisfy (by construction) the correct WI of (4.1).

The Ansatz of (4.4) mimics that of Eq. (3.8) to the extent that the first term contains the right structure to produce, when inserted into the first term on the rhs of (4.2), the derivative term appearing on the lhs of (3.12). The rhs of (3.12) is already there: it is the second term on the rhs of (4.2), originating directly from the seagull diagram $\left(a_{2}\right)$.

Similarly, a simple Ansatz for $V_{\mu \alpha \beta}$ that captures the two essential characteristics of having a (composite), longitudinally coupled poles, and satisfying the WI of (4.5) is

$$
V_{\mu \alpha \beta}=V_{\mu \alpha \beta}^{\ell}+V_{\mu \alpha \beta}^{\mathrm{t}}
$$

where

$$
V_{\mu \alpha \beta}^{\ell}=\frac{q_{\mu}}{q^{2}}\left[\widetilde{m}^{2}(k)-\widetilde{m}^{2}(k+q)\right] g_{\alpha \beta},
$$

and with the transverse part $V_{\mu \alpha \beta}^{\mathrm{t}}$ satisfying $q^{\mu} V_{\mu \alpha \beta}^{\mathrm{t}}=0$. We can write the vertex of (4.4) equivalently as

$$
i \widetilde{\Gamma}_{\mu \alpha \beta}=\left[\frac{\Delta^{-1}(k+q)-\Delta^{-1}(k)}{(k+q)^{2}-k^{2}}\right] \widetilde{\Gamma}_{v \alpha \beta}^{(0)}+\bar{V}_{\mu \alpha \beta},
$$


with

$$
\bar{V}_{\mu \alpha \beta}=V_{\mu \alpha \beta}+V_{\mu \alpha \beta}^{\mathrm{r}}
$$

where

$$
V_{\mu \alpha \beta}^{\mathrm{r}}=(2 k+q)_{\mu}\left[\frac{\widetilde{m}^{2}(k+q)-\widetilde{m}^{2}(k)}{(k+q)^{2}-k^{2}}\right] g_{\alpha \beta} .
$$

The term $V_{\mu \alpha \beta}^{\mathrm{r}}$ is a residual piece, acting as an additional (non-perturbative) vertex term, originating from forcing the vertex to assume the form of (4.8). This last way of writing $\widetilde{\Gamma}^{v \alpha \beta}$ makes the use of the basic identity of Eq.(3.12) immediate. Thus, after these rearrangements, we have that the final non-perturbative effective vertex $\bar{V}_{\mu \alpha \beta}$ must be transverse, $q^{\mu} \bar{V}_{\mu \alpha \beta}=0$.

Substituting for the $\widetilde{\Gamma}^{\mu \alpha \beta}$ on the rhs the expression given in (4.8) we obtain after simple algebra

$$
\Delta^{-1}\left(q^{2}\right)=q^{2}-\frac{i g^{2} C_{\mathrm{A}}}{2(d-1)}\left[\Pi(q)+\Pi_{\widetilde{m}}(q)\right]
$$

with

$$
\Pi(q)=(7 d-8) q^{2} \int_{k} \frac{\Delta(k+q)-\Delta(k)}{(k+q)^{2}-k^{2}}+4 d\left[\int_{k} k^{2} \frac{\Delta(k+q)-\Delta(k)}{(k+q)^{2}-k^{2}}+\frac{d}{2} \int_{k} \Delta(k)\right],
$$

and

$$
\Pi_{\widetilde{m}}(q)=\int_{k} \widetilde{\Gamma}_{\mu \alpha \beta}^{(0)} \Delta(k) \Delta(k+q)\left[V^{\ell}+\left\{V^{\mathrm{t}}+V^{\mathrm{r}}\right\}\right]^{\mu \alpha \beta} .
$$

The term in square brackets on the rhs of (4.12) has exactly the structure needed for employing (3.12). Note the perfect balance of relative coefficients required for this to happen! This becomes possible within the PT-BFM framework thanks to the special vertices appearing in the SDE; instead, in the conventional SD formulation (e.g., in the $R_{\xi}$ gauges) it would be very difficult to obtain the precise combination of terms needed for implementing (3.12). Evidently, by virtue of (3.12) it is clear that $\Pi(0)=0$. Thus, the part of the calculation determining $\Pi(q)$ is very similar to that of scalar QED, in the sense that it leads to total seagull annihilation, keeping the gluon massless.

On the other hand, the term $\Pi_{\widetilde{m}}(q)$, not present in the scalar QED study, makes it possible to have $\Delta^{-1}(0) \neq 0$ for the gluons. Assuming, for simplicity, that the dominant contribution in (4.13) comes from $V^{\ell}$, we obtain

$$
\Pi_{\widetilde{m}}(q)=-\frac{2 d}{q^{2}} \int_{k} \widetilde{m}^{2}(k) \Delta(k) \Delta(k+q)\left[(k+q)^{2}-k^{2}\right] .
$$

Now, in the limit $q^{2} \rightarrow 0$ (in Euclidean space) we have that

$$
\lim _{q^{2} \rightarrow 0}\left\{\frac{1}{q^{2}} \int_{k_{E}} \widetilde{m}^{2}(k) \Delta(k) \Delta(k+q)\left[(k+q)^{2}-k^{2}\right]\right\}=-\frac{1}{2} \int_{k_{E}} k^{2} \Delta^{2}\left(k^{2}\right)\left[\widetilde{m}^{2}\left(k^{2}\right)\right]^{\prime} .
$$

where the "prime" denotes differentiation with respect to $k^{2}$. Note that a monotonically decreasing mass, $\left[\widetilde{m}^{2}\left(k^{2}\right)\right]^{\prime}<0$, guarantees the positivity of $\widetilde{m}^{2}(0)$ (in Euclidean space)

An important consequence of this analysis is that Eq. (4.11) can be split unambiguously into two parts, one that vanishes as $q^{2} \rightarrow 0$ and one that does not. In fact, using (4.3) on the lhs of 
(4.11), we can assign the two types of contributions into two separate (but coupled) equations, namely (Minkowski space)

$$
\begin{aligned}
q^{2} H^{-1}(q) & =q^{2}-\frac{i g^{2} C_{\mathrm{A}}}{2(d-1)} \Pi(q), \\
\widetilde{m}^{2}(q) & =\frac{i g^{2} C_{\mathrm{A}}}{2(d-1)} \Pi_{\widetilde{m}}(q) .
\end{aligned}
$$

As we will see shortly, the first equation will determine the momentum dependence of the effective charge, and the second the running of the gluon mass.

\section{Effective charge and gluon mass: coupled but unique}

It is well-known that, due to the Abelian WIs of the PT-BFM Green's functions, the product

$$
\widehat{d}_{0}\left(q^{2}\right)=g_{0}^{2} \widehat{\Delta}_{0}\left(q^{2}\right)=g^{2} \widehat{\Delta}\left(q^{2}\right)=\widehat{d}\left(q^{2}\right)
$$

forms a renormalization-group ( $\mathrm{RG}$ )-invariant ( $\mu$-independent) quantity [1]. In order to realize Eq.(5.1) non-perturbatively, first set

$$
\widetilde{m}^{2}\left(q^{2}\right)=m^{2}\left(q^{2}\right) H^{-1}\left(q^{2}\right)
$$

where $m^{2}\left(q^{2}\right)$ is assumed to be the RG-invariant dynamical gluon mass. Then

$$
\widehat{\Delta}\left(q^{2}\right)=\frac{H\left(q^{2}\right)}{q^{2}+m^{2}\left(q^{2}\right)},
$$

and from the requirement that $g^{2} \widehat{\Delta}(q)$ must be RG-invariant we have that

$$
g^{2} H\left(q^{2}\right)=\bar{g}^{2}\left(q^{2}\right)
$$

Therefore, we finally arrive at the RG-invariant combination

$$
\widehat{d}\left(q^{2}\right) \equiv g^{2} \widehat{\Delta}\left(q^{2}\right)=\bar{g}^{2}\left(q^{2}\right) \bar{\Delta}\left(q^{2}\right),
$$

with

$$
\bar{\Delta}\left(q^{2}\right)=\frac{1}{q^{2}+m^{2}\left(q^{2}\right)}
$$

So, $\widehat{d}\left(q^{2}\right)$ is written as the product of two RG-invariant quantities: the dimensionless running coupling $\bar{g}^{2}\left(q^{2}\right)$ and the dimensionful "massive" gluon propagator $\bar{\Delta}\left(q^{2}\right)$.

We next cast our analysis in terms of the RG-invariant quantities defined above. The use of the spectral representation $[1,17]$ for $\Delta\left(q^{2}\right)$, namely

$$
\Delta\left(q^{2}\right)=\int d \lambda^{2} \frac{\rho\left(\lambda^{2}\right)}{q^{2}-\lambda^{2}+i \varepsilon}
$$



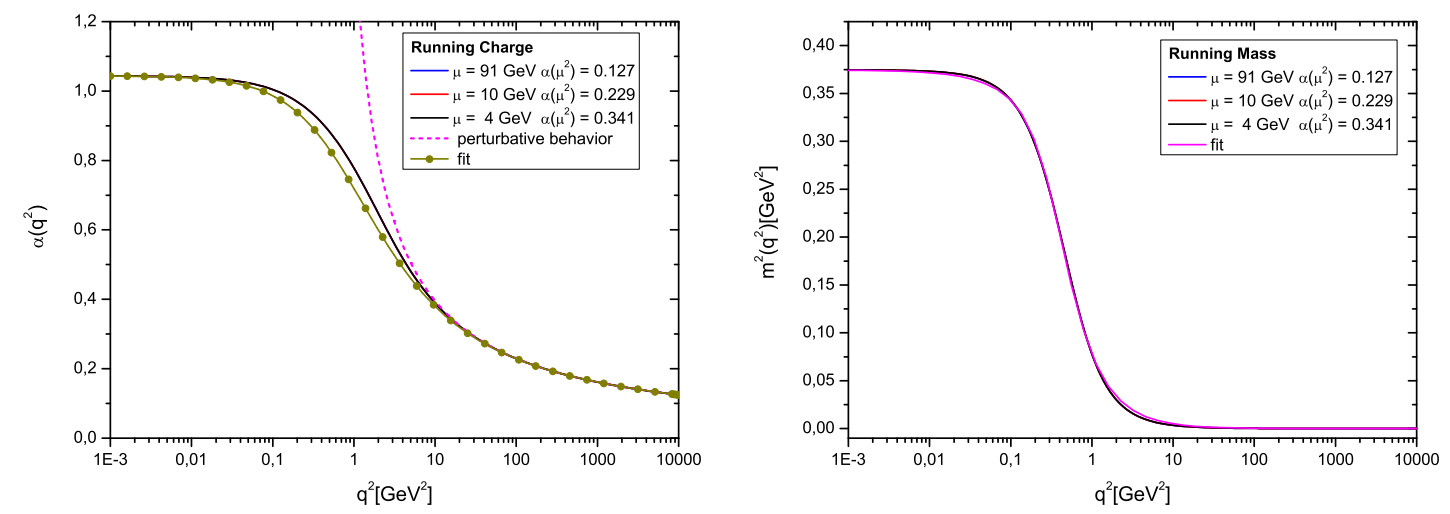

Figure 4: Numerical solutions displaying the momentum-dependence of the effective charge and the gluon mass, for three different values of the renormalization point $\mu$.

results in a spectacular simplification, because it "solves" the combination $\left\{\frac{\Delta(k+q)-\Delta(k)}{(k+q)^{2}-k^{2}}\right\}$ appearing in (4.12). After a series of standard assumptions one finally obtains [9]

$\frac{1}{\bar{g}^{2}\left(q^{2}\right)}=\frac{1}{\bar{g}^{2}\left(\mu^{2}\right)}+\tilde{b}\left[\int_{0}^{q^{2} / 4} d z\left(1+\frac{4 z}{5 q^{2}}\right)\left(1-\frac{4 z}{q^{2}}\right)^{1 / 2} \bar{\Delta}(z)-\int_{0}^{\mu^{2} / 4} d z\left(1+\frac{4 z}{5 \mu^{2}}\right)\left(1-\frac{4 z}{\mu^{2}}\right)^{1 / 2} \bar{\Delta}(z)\right]$,

and

$$
\frac{m^{2}\left(q^{2}\right)}{\bar{g}^{2}\left(q^{2}\right)}=\frac{2 \tilde{b}}{5}\left[\bar{\Delta}\left(q^{2}\right) \int_{0}^{q^{2}} d y y m^{2}(y) \bar{\Delta}(y)-\frac{1}{2} \int_{q^{2}}^{\infty} d y y^{2} \bar{\Delta}^{2}(y) \bar{g}^{2}(y)\left[m^{2}(y)\right]^{\prime}\right],
$$

where $\tilde{b}=10 C_{\mathrm{A}} / 48 \pi^{2}$; the discrepancy from the factor $b=11 C_{\mathrm{A}} / 48 \pi^{2}$, namely the first coefficient of the QCD one-loop $\beta$-function, is due to the (gauge-invariant!) omission of the ghost loops.

To study the behavior of the solutions of (5.9) for asymptotically large $q^{2}$, set $\bar{\Delta}(x) \rightarrow 1 / x$ and $\bar{\Delta}(y) \rightarrow 1 / y$ to arrive at

$$
m^{2}\left(q^{2}\right) \ln q^{2}=\frac{2}{5}\left[\frac{1}{q^{2}} \int_{0}^{q^{2}} d y m^{2}(y)-\frac{1}{2} \int_{q^{2}}^{\infty} d y \bar{g}^{2}(y)\left[m^{2}(y)\right]^{\prime}\right] .
$$

It is relatively straightforward to establish that the asymptotic solutions of (5.10) display power-law running. Indeed, substituting on both sides of (5.10) a $m^{2}\left(q^{2}\right)$ of the form

$$
m^{2}\left(q^{2}\right)=\frac{\lambda_{0}^{4}}{q^{2}}\left(\ln q^{2}\right)^{\gamma-1}
$$

one recognizes that the second term on the rhs of (5.11) is subleading, and that (5.11) is a solution of (5.10) provided that $\gamma=\frac{2}{5}$.

We next solve numerically the two coupled integral equations, renormalizing them at three different points, namely $\mu=\{4,10,91\} \mathrm{GeV}$, with $\alpha\left(\mu^{2}\right)=g^{2}(\mu) / 4 \pi=\{0.341,0.229,0.127\}$, respectively. In Fig. 4, we show the results for $\alpha\left(q^{2}\right)$ and $m^{2}\left(q^{2}\right)$; for either quantity we see clearly 


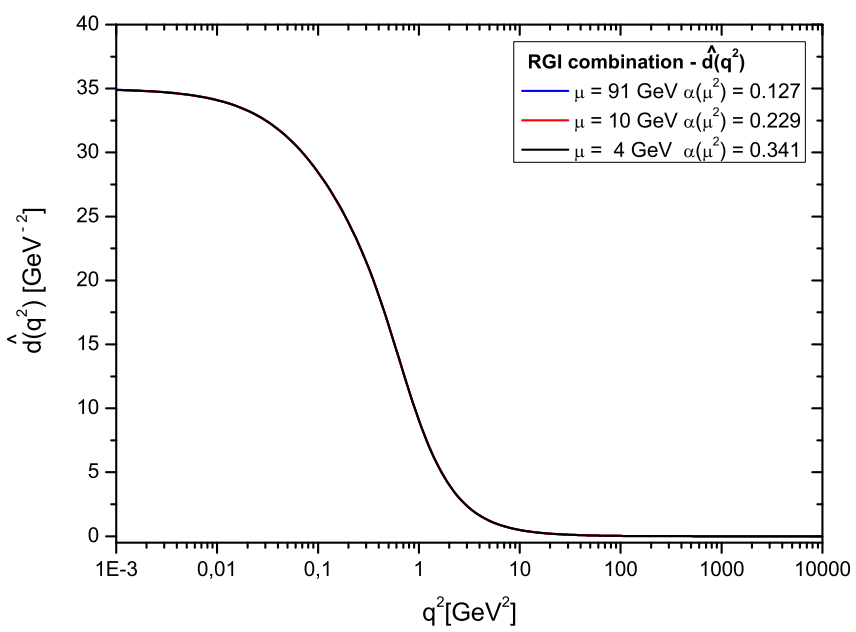

Figure 5: The RG-invariant product $\widehat{d}\left(q^{2}\right)$ obtained by combining the results for $\alpha\left(q^{2}\right)$ and $m^{2}\left(q^{2}\right)$.

that the three curves merge practically into a single one, thus confirming numerically their $\mu$ independence, expected on formal grounds. The solutions for $\alpha\left(q^{2}\right)$ may be fitted by the physically motivated functional form [1], namely

$$
\alpha\left(q^{2}\right)=\frac{1}{4 \pi \tilde{b} \ln \left[\left(q^{2}+t m_{0}^{2}\right) / \Lambda^{2}\right]},
$$

with $t=3.7$ and $\Lambda=645 \mathrm{MeV}$. The behavior of $m^{2}\left(q^{2}\right)$ in the entire range of momenta can be accurately described by the following parametrization

$$
m^{2}\left(q^{2}\right)=\frac{m_{0}^{4}}{q^{2}+m_{0}^{2}}\left[\ln \left(\frac{q^{2}+f\left(q^{2}, m_{0}^{2}\right)}{\Lambda^{2}}\right) / \ln \left(\frac{f\left(0, m_{0}^{2}\right)}{\Lambda^{2}}\right)\right]^{-3 / 5},
$$

where $f\left(q^{2}, m_{0}^{2}\right)=\rho_{1} m_{0}^{2}+\rho_{2} \frac{m_{0}^{4}}{q^{2}+m_{0}^{2}}$, with $\rho_{1}=-1 / 2, \rho_{2}=5 / 2$, and $m_{0}=612 \mathrm{MeV}$. Finally, with the help of Eq. (5.5) we can construct $\widehat{d}\left(q^{2}\right)$ out of the numerical solutions for $\alpha\left(q^{2}\right)$ and $m^{2}\left(q^{2}\right)$; the result is shown in Fig. 5. Obviously, since $\widehat{d}\left(q^{2}\right)$ is built out of two quantities that are individually independent of $\mu$, it too turns out to be $\mu$-independent; this property is clearly observed in Fig. 5 .

\section{Conclusions}

The analysis presented here demonstrates that the appearance of seagull divergences in gluon mass generation is caused by a subtle mismatch between two parallel field theoretic mechanisms. Specifically, the Schwinger mechanism, which requires the appearance of massless poles in the three-gluon vertex, distorts the mechanism responsible for the cancellation of the seagull divergences, unless the poles enter into the gluon vertex in a very particular way. A concrete example of a vertex that does not produce any clash between these two mechanism has been given, and the implications for the resulting SDE have been worked out. The elimination of the seagull divergences allows the resulting SDE equation to be separated unambiguously into two distinct dynamical equations, determining the gluon mass and the QCD effective charge. This, in turn, is 
a significant improvement over the existing approaches, where the infrared behavior of these two quantities had to be extracted (not without a certain ambiguity) from $\widehat{d}\left(q^{2}\right)$. It is clear that the methodology outlined here should be applied to gauges where the results can be directly compared to lattice simulations (such as the Landau gauge). Given that the solutions for $\alpha\left(q^{2}\right)$ and $m^{2}\left(q^{2}\right)$, and therefore for $\widehat{d}\left(q^{2}\right)$ and/or $\Delta\left(q^{2}\right)$, are expected to be unique, one should be able to test if the freezing value $\Delta(0)$ obtained within this new SDE treatment coincides with that seen on the lattice. We hope to report progress in this direction in the near future.

Acknowledgments:

I would like to thank the ECT* for making the QCDTNT workshop possible. This research was supported by the European FEDER and Spanish MICINN under grant FPA2008-02878, the program Prometeo/2009/129 (Generalitat Valenciana), and the Fundación General of the UV.

\section{References}

[1] J. M. Cornwall, Phys. Rev. D 26, 1453 (1982).

[2] A. C. Aguilar and J. Papavassiliou, JHEP 0612, 012 (2006).

[3] A. C. Aguilar, D. Binosi and J. Papavassiliou, Phys. Rev. D 78, 025010 (2008).

[4] J. M. Cornwall and J. Papavassiliou, Phys. Rev. D 40, 3474 (1989); A. Pilaftsis, Nucl. Phys. B 487, 467 (1997); D. Binosi and J. Papavassiliou, Phys. Rev. D 66(R), 111901 (2002); Phys. Rept. 479, 1 (2009); V. P. Nair, "Quantum field theory: A modern perspective," New York, USA: Springer (2005) $557 \mathrm{p}$.

[5] L. F. Abbott, Nucl. Phys. B 185, 189 (1981).

[6] A. Cucchieri and T. Mendes, PoS LAT2007, 297 (2007); Phys. Rev. Lett. 100, 241601 (2008); arXiv:0904.4033 [hep-lat].

[7] I. L. Bogolubsky et al., PoS LATTICE, 290 (2007); P. O. Bowman et al., Phys. Rev. D 76, 094505 (2007); T. Iritani, H. Suganuma and H. Iida, arXiv:0908.1311 [hep-lat]; O. Oliveira and P. J. Silva, arXiv:0910.2897 [hep-lat].

[8] C. Alexandrou, P. de Forcrand and E. Follana, Phys. Rev. D 63, 094504 (2001); F. D. R. Bonnet et al., Phys. Rev. D 62, 051501 (2000); F. D. R. Bonnet et al., Phys. Rev. D 64, 034501 (2001).

[9] A. C. Aguilar and J. Papavassiliou, arXiv:0910.4142 [hep-ph].

[10] J. S. Schwinger, Phys. Rev. 125, 397 (1962); Phys. Rev. 128, 2425 (1962).

[11] R. Jackiw and K. Johnson, Phys. Rev. D 8, 2386 (1973); J. M. Cornwall and R. E. Norton, Phys. Rev. D 8 (1973) 3338; E. Eichten and F. Feinberg, Phys. Rev. D 10, 3254 (1974).

[12] J. S. Ball and T. W. Chiu, Phys. Rev. D 22, 2542 (1980).

[13] D. Binosi and J. Papavassiliou, Phys. Rev. D 77, 061702 (2008); JHEP 0811, 063 (2008)

[14] P. A. Grassi, T. Hurth and M. Steinhauser, Annals Phys. 288, 197 (2001); D. Binosi and J. Papavassiliou, Phys. Rev. D 66, 025024 (2002).

[15] M. Lavelle, Phys. Rev. D 44, 26 (1991); A. C. Aguilar and J. Papavassiliou, Eur. Phys. J. A 35, 189 (2008); D. Dudal et al., Phys. Rev. D 78, 065047 (2008).

[16] D. Binosi, arXiv:0911.0315 [hep-ph].

[17] J. M. Cornwall, arXiv:0904.3758 [hep-ph]. 